\title{
Persistence of Multiple Interaction Paths for Individual Inter-Area Modes *
}

\author{
Yuwa Chompoobutrgool* Luigi Vanfretti ${ }^{*}$ \\ ${ }^{*}$ KTH Royal Institute of Technology, School of Electrical Engineering, \\ Stockholm, Sweden (E-mail: yuwa@ee.kth.se, luigiv@kth.se).
}

\begin{abstract}
Dominant interaction paths are the passageways containing the highest modal content of particular oscillations in interconnected power systems. For the case of inter-area modes, pinpointing the dominant path that corresponds to each mode may allow for the extraction of signals that bear a significant modal content at a particular inter-area frequency. Strong modal content for these inter-area modes may also be spread onto secondary paths. These secondary paths bear similar properties as those shown by dominant paths. The aim of this study is thus to demonstrate the persistence of both dominant and secondary paths by employing "network modeshape" properties. The existence of the paths is verified by nonlinear time-domain simulations where modal content at each inter-area frequency is extracted. The proper selection of feedback signals from the dominating paths may increase the flexibility of WAPODs by providing a number of signal choices to be used as backup in the presence of contingencies.
\end{abstract}

Keywords: Inter-area oscillations; sensitivity analysis; interaction paths; wide-area power oscillation damper (WAPOD).

\section{INTRODUCTION}

One of PMU-data based applications of wide-area monitoring systems (WAMS) that has received much attention recently is to exploit existing and potential remote signals from phasor measurement units (PMUs) [Kamwa et al. (2006)] for inter-area mode control. Wide-area power oscillation dampers (WAPOD) may be used to damp oscillations in large interconnected networks [N.R. Chaudhuri, et al (2010)].

An important issue regarding the use of WAPOD for enhancing damping of low-frequency inter-area oscillations (LFIOs) is feedback signal selection. The question is, in large and complex interconnected power systems, which signal(s) gives the best damping of such modes, considering signal availability. Presumably, these signals have the highest content of the mode of interest, and are the best candidates for feedback control. This article continues an investigation on the question of where are these signals located within the system, and how they can be generated.

In a prior study Chompoobutrgool and Vanfretti (2012), we have investigated the passageways having the highest inter-area modal contents, i.e., main interaction paths. The principle of the study is that for each individual mode of oscillation, the propagation paths and their origin are deterministic and distinctive. Among propagation paths, there exists only one path which contains the largest portion of such mode; namely, dominant path. In the study, the persistence of the dominant inter-area path

\footnotetext{
* Invited Paper - Special Session: "Stability and Control in Smart Power Grid", 8th IFAC Symposium on Power Plants and Power Systems (PP\&PSC), 2012.

Y. Chompoobutrgool is supported by Elforsk, Sweden.

Luigi Vanfretti is supported by the STandUP for Energy collaboration
} initiative and the KTH School of Electrical Engineering. was verified and the signals obtained from the path were analysed. In addition, main features of the dominant path were described.

In continuation from this previous study, this article further investigates the second strongest inter-area interaction path, namely, secondary path. Although having a smaller modal content, these secondary paths bear similar properties as those shown by dominant paths. The aim of this study is thus to demonstrate the persistence of both dominant and secondary paths by employing "network modeshape" properties. The existence of the paths is verified by nonlinear time-domain simulations where modal content at each inter-area frequency is extracted. Using these results, the article proposes to construct signals from the dominant and secondary paths or a combination from both paths as candidates to be used as feedback control signals. The proper selection of feedback signals from dominant or secondary paths (or their combination) may increase the flexibility of WAPODs by providing a number of signal choices to be used as backup in the presence of contingencies, which could be communication failures.

\section{BASIS OF STUDY}

\subsection{Interaction Paths}

The concept of "interaction paths" was used to characterize the dynamic behavior of the Western Electric Coordinating Council (WECC) power system in [Hauer et al. (2007)] where the interaction paths are defined as the group of transmission lines, buses, and controllers which the generators in a system use for exchanging energy during swings. Experiences through extensive signal analysis shows that there exists coherency in the oscillatory content of key signals within these interaction paths. This approach allows for tracking the transmission corridors with the oscillatory content of interest. 
Building from these observation, we propose to establish a methodical process to characterize such paths in the prequel study [Chompoobutrgool and Vanfretti (2012)] where the concept of "dominant inter-area paths" is introduced. These dominant paths are defined as the passageways containing the highest content of the inter-area oscillations. The propagation of the oscillatory modes in the paths on interconnected power systems is deterministic and unique; i.e., each oscillatory mode has its own distinct travelling paths under a certain operating condition. Distinct features of the paths and their corresponding persistence are provided and evaluated in the study. The presumption is that signals from these paths are the most observable and have, to some extent, high degree of robustness among other signals within a system since they have the highest content of inter-area modes. Hence, these signals are prospective candidates as feedback input signals to WAPODs.

\subsection{Network Modeshape, $S$}

The notion of the dominant and secondary paths can be quantitatively expressed by the product of the two key factors: mode shapes belonging to electromechanical oscillations and sensitivities of network variables. This relationship is termed "network modeshape" which is introduced in [Vanfretti (2009); Vanfretti and Chow (2010)]. It indicates how much of the content of each mode ${ }^{1}$ is distributed within the network variables. In other words, the larger in magnitude the network modeshape is, the more observable the signals measured from the path become. Because WAMS provide singals measured by PMUs, we focus on those whose synchrophasors are directly measured. The variables of interest are voltage magnitude and voltage angles; thus, only their corresponding modeshapes $\left(S_{V}\right.$ and $\left.S_{\theta}\right)$ are considered in this study.

Consider a linearized $N$-machine system in a state-space form

$$
\begin{aligned}
& \Delta \dot{x}=A \Delta x+B \Delta u \\
& \Delta y=C \Delta x+D \Delta u
\end{aligned}
$$

where vectors $\Delta x, \Delta y$, and $\Delta u$ represent the state variables, the output variables and the inputs, respectively. With no input, the electromechanical model is expressed as

$$
\underbrace{\left[\begin{array}{c}
\Delta \dot{\delta} \\
\Delta \dot{\omega}
\end{array}\right]}_{\Delta \dot{x}}=\underbrace{\left[\begin{array}{ll}
A_{11} & A_{12} \\
A_{21} & A_{22}
\end{array}\right]}_{A} \underbrace{\left[\begin{array}{c}
\Delta \delta \\
\Delta \omega
\end{array}\right]}_{\Delta x}
$$

where matrix $A$ represent the state matrix corresponding to the state variables $\Delta \delta$ and $\Delta \omega$. Then, performing eigenanalysis, the electromechanical mode shape is derived from

$$
A W(A)=\lambda W(A)
$$

where $\lambda$ are eigenvalues of the electromechanical modes of the system and $W(A)$ are the right eigenvectors or mode shape that is used to compute the network modeshape.

Using $C$ from (1), sensitivities of the voltage magnitude $\left(C_{V}\right)$ and voltage angle $\left(C_{\theta}\right)$ are expressed as

\footnotetext{
1 The focus here and in (Chompoobutrgool and Vanfretti, 2012) is on inter-area modes, however the theory is applicable to any mode.
}

$$
\underbrace{\left[\begin{array}{c}
\Delta V \\
\Delta \theta
\end{array}\right]}_{\Delta y}=\underbrace{\left[\begin{array}{ll}
\frac{\partial \Delta V}{\partial \Delta \delta} & \frac{\partial \Delta V}{\partial \Delta \omega} \\
\frac{\partial \Delta \theta}{\partial \Delta \delta} & \frac{\partial \Delta \theta}{\partial \Delta \omega}
\end{array}\right]}_{C} \underbrace{\left[\begin{array}{c}
\Delta \delta \\
\Delta \omega
\end{array}\right]}_{\Delta x}=\left[\begin{array}{ll}
C_{V} & C_{\theta}
\end{array}\right]^{T}\left[\begin{array}{c}
\Delta \delta \\
\Delta \omega
\end{array}\right]
$$

Then, the expressions for voltage magnitude and voltage angle modeshapes $\left(S_{V}\right.$ and $\left.S_{\theta}\right)$ are

$$
S_{V}=C_{V} W(A), \quad S_{\theta}=C_{\theta} W(A) .
$$

Important Features of Dominant Inter-Area Paths

- The largest $S_{V}$ or the smallest $S_{\theta}$ element(s) indicates the center of the path. This center can be theorized as the "inter-area mode center of inertia" or the "interarea pivot" for each of the system's inter-area modes.

- The difference between $S_{\theta}$ elements of two edges of the path are the largest among any other pair within the same path. In other words, the oscillations are the most positive at one end while being the most negative at the other end. Hence, they can be theorized as the "tails" for each inter-area mode.

- $S_{V}$ elements of the edges are the smallest or one of the smallest within the path.

In extension to the previous study, another concept of interaction paths is introduced in this article: "secondary inter-area paths". They are defined as the passageways containing the second highest (to the dominant path) inter-area mode content. These secondary paths share, though not completely, the same characteristics as those of the dominant paths. The persistence of these secondary paths will be examined in the next section.

\section{STUDY SYSTEM AND INTERACTION PATHS}

\subsection{Study System Description}

The system under study, namely KTH-NORDIC32 (see Fig. 1), is a conceptualization of the Swedish power system and its neighbors circa 1995; it was constructed from the power system data proposed by T. Van Cutsem [T. Van Cutsem (2010)] and further modified in [Chompoobutrgool et al. (2012)]. It has 20 generators, 12 of which are hydro generators located in the North and Equivalent areas, whereas the rest are thermal generators located in the Central and South areas. The northern regions (the North and Equivalent) account for most of the generation causing a heavy power transfer to the southern regions (the Central and South) through weak ties. As a result, this looselyconnected system exhibits lightly damped low frequency inter-area oscillations. The two with the lowest damping ratios are of major concern in this study and will be discussed in the following section.

Two loading scenarios are considered: heavy flow and moderate flow. Power generation and consumptions for each scenario are summarized in Table 1. Note that to develop a fundamental understanding, controls such as exciters and turbine governors are removed, and the system has no damping. Nevertheless, comprehensive and detailed modelling of this test system considers controllers as described in Chompoobutrgool et al. (2012).

\subsection{Small Signal Analysis}

Small-signal stability is defined as the ability of a power system to maintain its synchronism after being subjected 
Table 1. Loading Scenarios.

\begin{tabular}{|l||l|l|}
\hline Flow Scenario & Heavy $(M W)$ & Moderate $(M W)$ \\
\hline \hline Total Generation & $11,304.41$ & $8,887.23$ \\
\hline Total Loads & $10,790.26$ & $8,757.1$ \\
\hline North to Central Flow & $3,183.79$ & $1,799.97$ \\
\hline
\end{tabular}

to a small disturbance Kundur and et al. (2004). Smallsignal stability analysis reveals important relationships among state variables of a system and gives an insight into the electromechanical dynamics of the network.

Applying the eigenanalysis to the linearized model of the KTH-NORDIC32 system, small-signal stability is studied by computing four properties: eigenvalues, frequency of oscillation, damping ratios and eigenvectors (or mode shapes). Table 2 shows the two lowest damping modes, their corresponding frequencies and damping ratios for each loading scenario. Although not shown here, the mode shapes reveal that Mode 1 for both scenario is the swing of the North and Equivalent machines against the Central and South machines while Mode 2 is the swing of the Central machines against the Equivalent and South machines. In both scenarios, damping ratios of Mode 1 and Mode 2 are insufficiently low and, thus, additional damping is necessary.

\subsection{Main Interaction Paths of the Study System}

The dominant and secondary inter-area paths of the interarea modes are illustrated in Fig. 1 where the star symbols denote dominant paths and the green cross symbols denote secondary paths. Mode 1 and Mode 2 are represented in yellow and green color, respectively. Voltage magnitude and angle modeshapes from both modes on both paths are depicted and compared between the two loading scenarios in Fig. 2 and 3. In these figures, blue dots indicate network modeshapes of the heavy flow while red dots indicate network modeshapes of the moderate flow.

Analyzing both figures, although there are significant drops in the voltage magnitude modeshapes in both paths when the loading scenario shifts from heavy to moderate, the characteristics of the dominant paths are preserved in Fig. 2(a), 2(b), and 3(a), this is a characteristic of secondary paths that are directly parallel to dominant paths Vanfretti and Chow (2009). However, the behavior seen in Fig. 3(b) is the characteristics of "extended paths", observe that similarly than in Vanfretti and Chow (2009), dominant paths will set the upper value of those buses which are connected to secondary paths and their value will decrease following the pseudo-travelling wave shape of dominant paths. Voltage angle modeshapes, on the other hand, preserve the shape of the dominant path and maintain their strength in both dominant and secondary paths; this is because they are following the of the "interarea mode center of inertia" Chompoobutrgool and Vanfretti (2012) between the clustered masses at the edges of each path. This result must be taken into account when choosing types of signals that require high observability.

\section{PERSISTENCE OF SECONDARY INTER-AREA OSCILLATION PATHS}

In continuation from the previous study in Chompoobutrgool and Vanfretti (2012), the same contingency scenarios are implemented on the test system: removal of the transmission lines that are in connection with the dominant

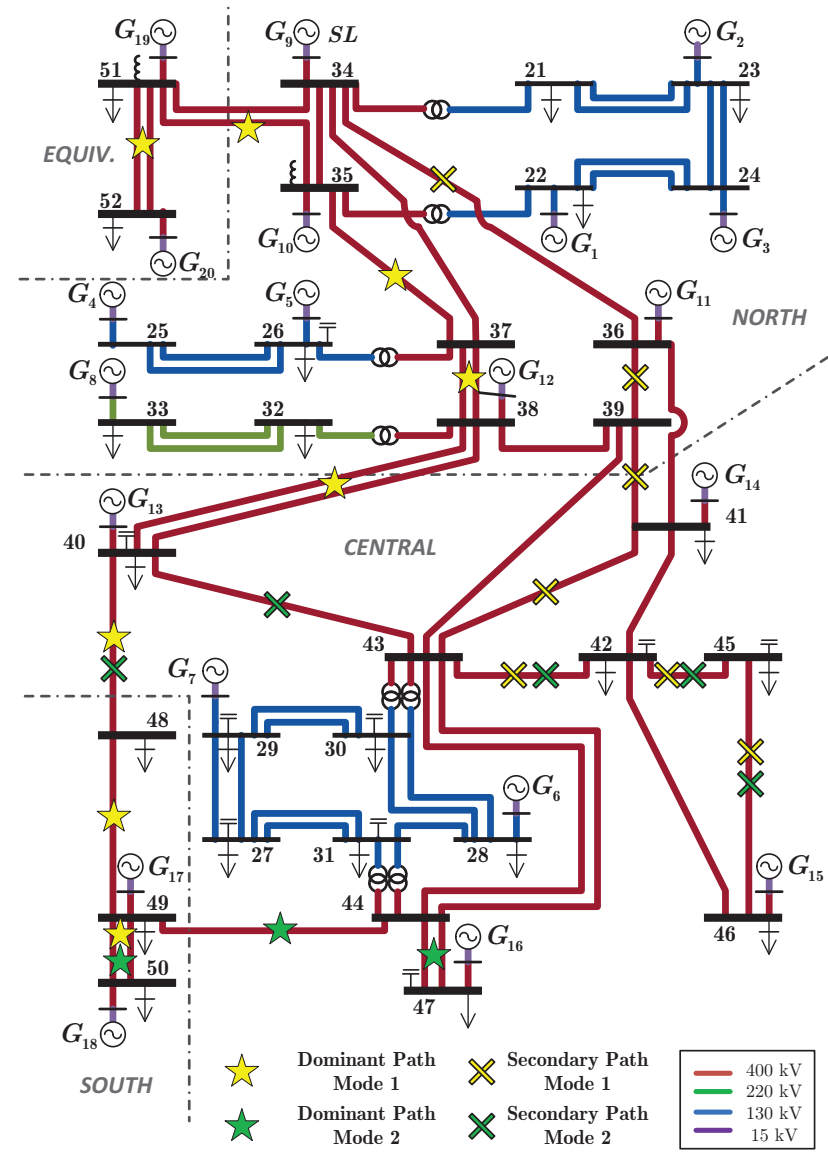

Fig. 1. Dominant inter-area paths of the heavy flow scenario: Mode 1 (yellow stars) and Mode 2 (green stars).

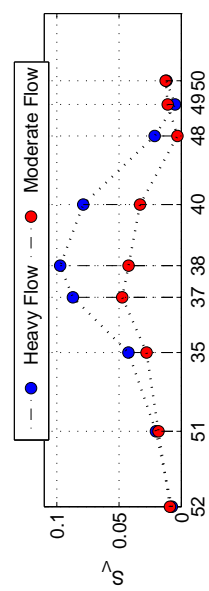

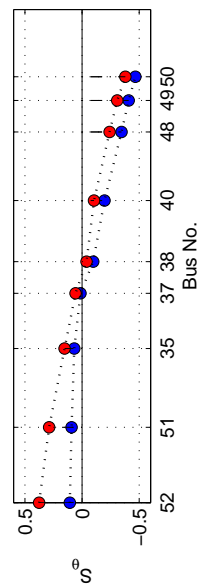

(a) Dominant Path.

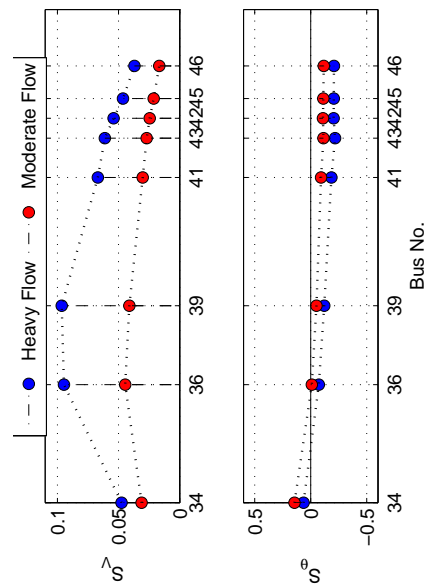

(b) Secondary Path.
Fig. 2. Voltage magnitude and angle modeshapes: Mode 1.

path of Mode 1 (0.49-Hz mode). The corresponding network modeshapes of Mode 1's secondary paths are calculated for each contingency to determine signal persistence: i.e., robustness and strength. The $x$-axis represents the bus number on the secondary paths; the distance between buses are proportional to the line impedance magnitude. The $y$-axis of the upper plot represents voltage magnitude modeshape $\left(S_{V}\right)$ while the lower plot represents voltage angle modeshape $\left(S_{\theta}\right)$ of the secondary inter-area paths. For every scenario, the removal of corridors are compared 
Table 2. Linear analysis results of the two lowest damping modes in KTH-NORDIC32

\begin{tabular}{|c||c|c|c|c|}
\hline Mode & Loading Scenario & Eigenvalues & Frequency $(\mathrm{Hz})$ & Damping ratio $(\%)$ \\
\hline \hline \multirow{2}{*}{1} & Heavy & $-0.0679 \pm j 3.0429$ & 0.4843 & 2.23 \\
& Moderate & $-0.1033 \pm j 3.7613$ & 0.5986 & 2.75 \\
\hline \multirow{2}{*}{2} & Heavy & $-0.1083 \pm j 4.4932$ & 0.7151 & 2.41 \\
& Moderate & $-0.2311 \pm j 4.88967$ & 0.7793 & 4.71 \\
\hline
\end{tabular}
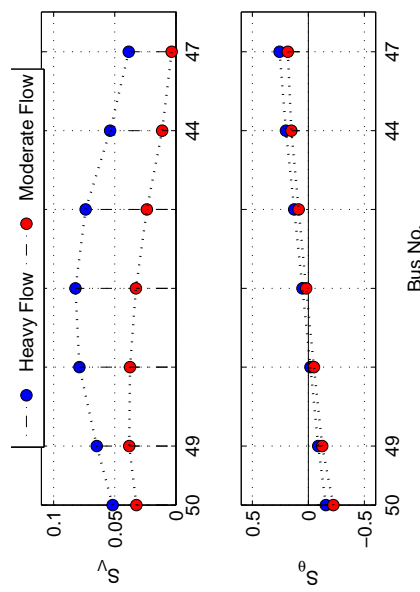

(a) Dominant Path.

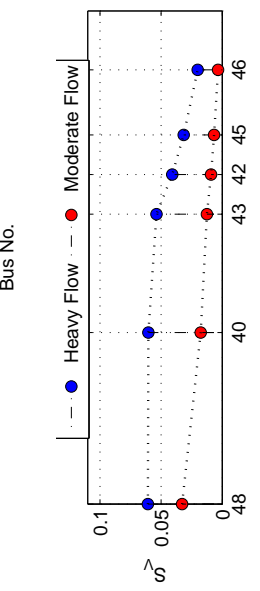

(b) Secondary Path

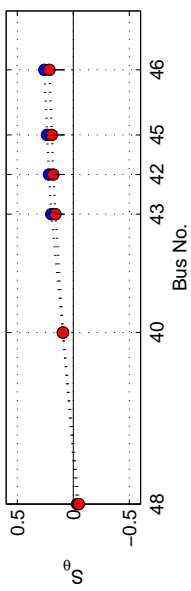

Fig. 3. Voltage magnitude and angle modeshapes: Mode 2.

to the nominal system (moderate flow and no lines lost) denoted by black dots to determine the persistence of the path. Note that only selected results will be shown in this paper.
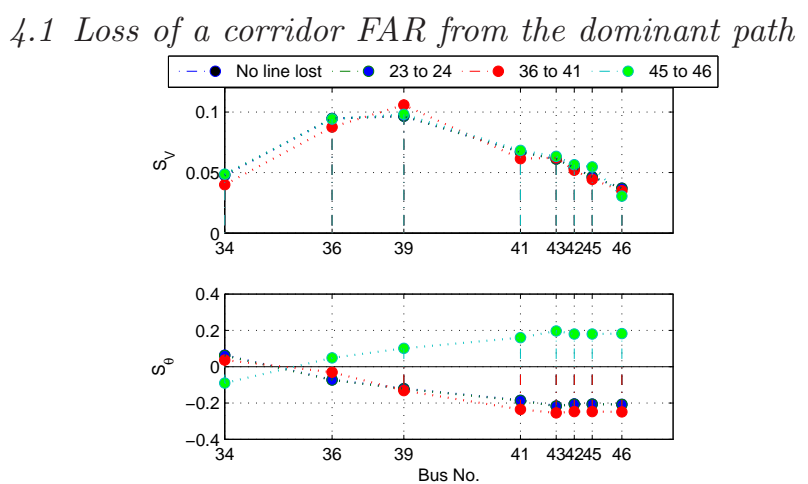

Fig. 4. $S_{V}$ and $S_{\theta}$ of the secondary path: Loss of a corridor

FAR from the dominant path.

Figure 4 shows the three selected corridors: 23-24, 36-41, and 45-46, which are located the farthest from the dominant path. For all cases, the results show that the voltage magnitude modeshapes $\left(S_{V}\right)$ remain consistent both in magnitude and direction. However, although having nearly similar magnitude to the nominal case, the voltage angle oscillations $\left(S_{\theta}\right)$ have opposite directions when corridor 45-46 is disconnected.

\subsection{Loss of a corridor NEAR the dominant path}

Figure 5 shows the three selected corridors: 38-39, 40-43, and 44-49, which are directly connected to the dominant path. Despite the sign change in $S_{\theta}$ with the disconnection of corridor 38-39, the removals of the first two corridors have trivial impact on the voltage modeshapes in terms of magnitude. However, it can be observed that the removal
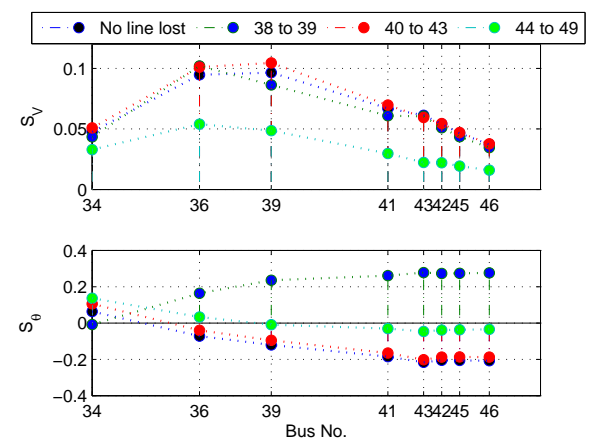

Fig. 5. $S_{V}$ and $S_{\theta}$ of the secondary path: Loss of a corridor NEAR the dominant path.

of corridor 44-49 results in a significant reduction in $S_{V}$ and $S_{\theta}$ in which the resulting magnitude is relatively small comparing to those of the other corridor removals.

\subsection{Loss of a corridor ON the dominant path}
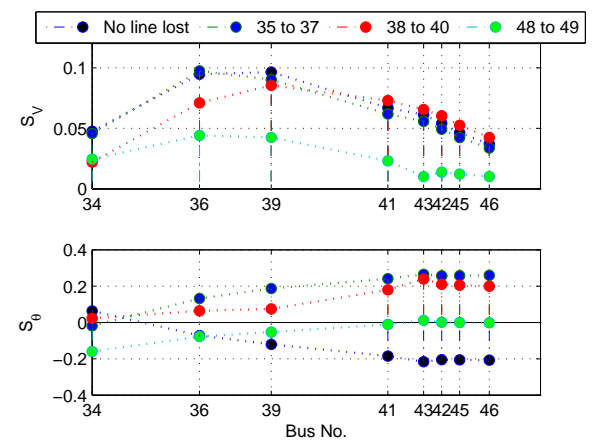

Fig. 6. $S_{V}$ and $S_{\theta}$ of the secondary path: Loss of a corridor ON the dominant path.

Figure 6 shows the three selected corridors: 35-37, 38-40, and 48-49, which are on the dominant path. Despite the sign change in $S_{\theta}$, the removals of the first two corridors have small impact on the voltage modeshapes in terms of magnitude. On the other hand, it can be observed that the removal of corridor 48-49 has a similar but more severe impact on both $S_{V}$ and $S_{\theta}$ of the secondary path comparing to the removal of corridor 44-49. Note that $S_{\theta}$ of the buses $41,43,42,45$ and 46 are difficult to observe.

\section{Discussion}

When the corridors connecting Bus 49 are disconnected, detrimental changes on the modeshapes are observed as in the case of corridor 44-49 or 48-49 being removed. Although not shown here, the removal of corridor 4950 results in the transition of the $0.49-\mathrm{Hz}$ mode to a new mode. This confirms that the origin of Mode 1 is from $G_{18}$ in which, from the small signal analysis, its speed variable is the most associated state of the mode. In nearly all of the contingency studies, the secondary path is persistent; i.e., the signals from the path endure 
their strength and visibility; hence robustness. Although there are cases with sign change in the voltage angle modeshapes, the magnitude remain closed to that of the nominal case. This sign change is due to a reversal in the direction of their corresponding mode shapes.

\section{PROPOSED SIGNALS AND THEIR JUSTIFICATION}

Using the results in the previous section, signals from the secondary path to be used as feedback inputs are proposed and justified here. Note that only the results of voltage angles are displayed and that latencies are neglected here (but will be considered in another study). Block diagram for the feedback signals using voltage angles is illustrated in Fig. 7.

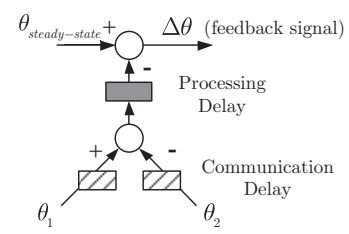

Fig. 7. Block diagram for constructing feedback signals using voltage angles.

To justify the selection, a small perturbation of 0.01 p.u. is applied to the linearized test system at the mechanical power of $G_{6}$ at $t=2 \mathrm{~s}$ for $1 \mathrm{~s}$, and the time responses of the selected outputs are simulated for $20 \mathrm{~s}$ and analyzed. To implement the network modeshape concept, the partial multimodal decomposition framework [Wei (2002)] is employed. With this approach, the network modeshape behaves as a filter allowing only the mode of interest (Mode 1 in this case) to be evaluated. (See more details in [Chompoobutrgool and Vanfretti (2012)]).

The observed responses are the deviation in the voltage angle whereby the bus voltage angle $\theta_{34}$ is used as a reference. Figure 8 (top) shows the simulated responses of the secondary path while the bottom figure shows the results of the responses after filtering through network modeshapes.

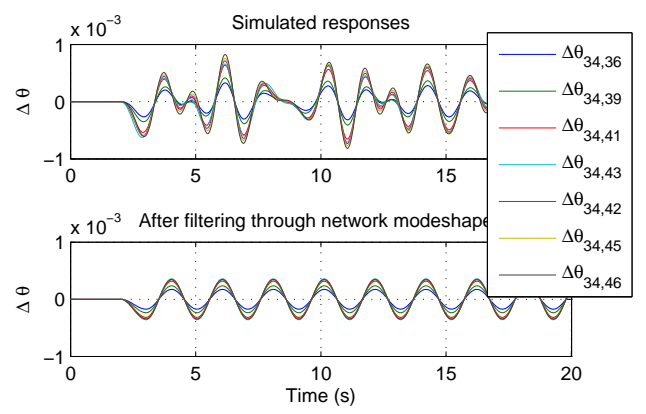

Fig. 8. $\Delta \theta$ responses of the secondary path signals after a perturbation at $G_{6}$.

Comparing the top to the bottom figures, the network modeshape filter not only extracts one mode from the rest, but also projects the proportion of the modal content within the signals. That is the true inter-area mode content that should be expected from the resulting signals.
The study in prequel demonstrates the angle combination of the dominant path while that of the secondary path is shown here. Although the signals from the dominant path are preferable to the secondary path's, a combination from both paths may offer another alternative if a communication network on the dominant path is lost. Figure 9 illustrates the voltage angle responses of signal combination between signals from the dominant path $\theta_{37}$ and from the secondary path $\theta_{39}, \theta_{43}$, and $\theta_{45}$. The results are compared with the response of only dominant path signals $\Delta \theta_{37,48}$ shown in the dashed line.

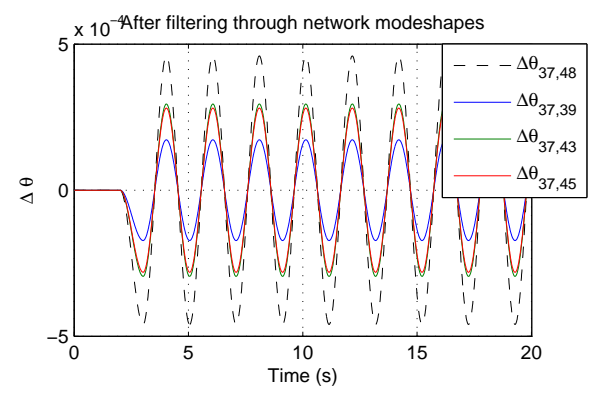

Fig. 9. $\Delta \theta$ responses of the signal combination after a perturbation at $G_{6}$.

Observe that the amplitude of each signal is proportional to the network modeshape of Mode 1 as shown in Fig. 2. As can be expected, the response of the pair from the dominant path, $\Delta \theta_{37,48}$, is the most observable among the others. Nevertheless, in the absence of the signal from Bus 48, the signal combination between the two paths provide sufficient modal content which can be used as feedback inputs to damping controllers.

\section{CONCEPT VALIDATION THROUGH NONLINEAR TIME-DOMAIN SIMULATION}

The concept of network modeshapes is derived from linear analysis and used to determine interaction paths. As such, time-domain simulation is employed to verify the dominant and secondary inter-area mode interaction action paths concept presented earlier, in the nonlinear framework. This approach is useful, the time-domain signals used can be considered as measurements from PMUs, making the approach applicable using synchrophasors. By implementing a small perturbation, the modal contents of interest are extracted from the responses and the dominant and secondary inter-area paths are reconstructed. Note that only the dominant and secondary paths of Mode 1, heavy flow scenario are considered here.

\subsection{Time-Domain Simulation Responses}

A perturbation of 0.1 p.u. is applied at the mechanical power of $G_{6}$ at $t=2 \mathrm{~s}$ for $1 \mathrm{~s}$ and the system is simulated for $20 \mathrm{~s}$. Responses of the voltage magnitude deviation of the buses on the dominant path are illustrated in Fig. 10(a) (top). From the simulated signals, the frequency component between range 0.48 and $0.50 \mathrm{~Hz}$ are extracted as shown in the bottom figure. Figure 10(b) shows the responses of the voltage angle deviation of the same buses after the extraction. The red dash line indicates the snapshot instant at which the corresponding magnitudes are taken to reconstruct the paths in the following section. 


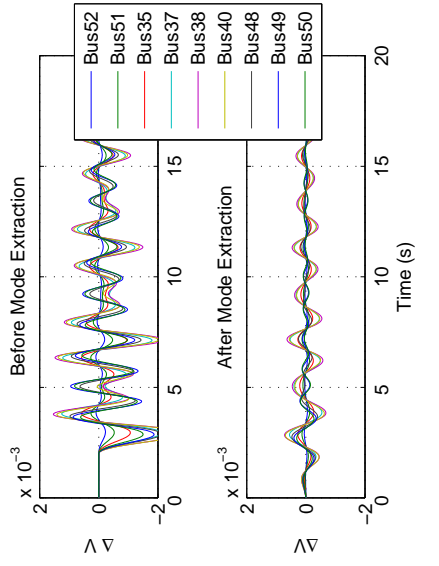

(a) Voltage Magnitude Deviation

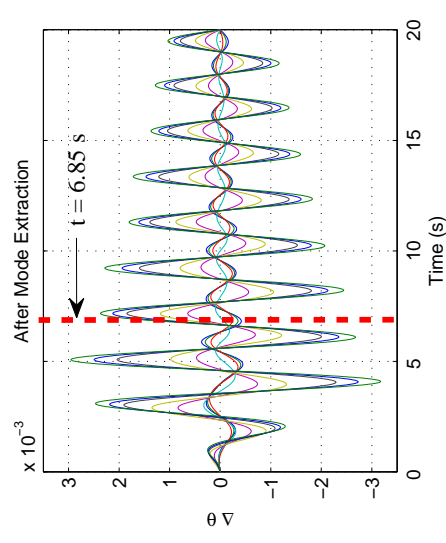

(b) Voltage Angle Deviation
Fig. 10. Responses after a perturbation of the signals on the dominant path of Mode 1.

\subsection{Reconstruction of the Interaction Paths from the} Nonlinear Simulations

Taking a snapshot at $\mathrm{t}=6.85 \mathrm{~s}$ (from Fig. 10(b)), the magnitude of each signal on the dominant and secondary paths of Mode 1 is plotted along the line impedance. The results are compared to those from the linear analysis as shown in Fig. 11 where the red dots refer to the nonlinear time-domain responses and the blue dots refer to the linear analysis in Section 3 (see Fig. 2).

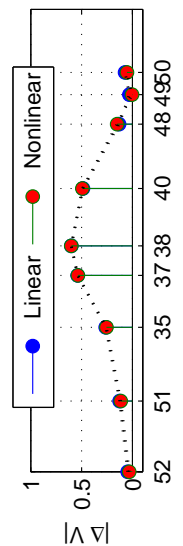

(a) Dominant Path.
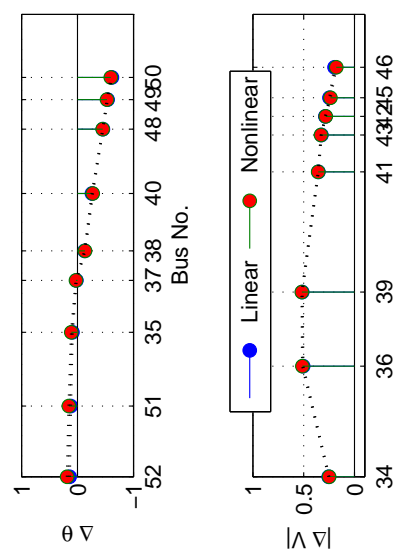

(b) Secondary Path
Fig. 11. Comparison between linear vs. nonlinear results: Mode 1.

The nonlinear results nearly duplicate those of the linear analysis; hence, the network modeshapes and inter-area mode dominant and secondary path concepts are verified. In any case, depending on the selected snapshot, the values may vary but the overall behavior bears the features of the dominant and secondary inter-area paths. A future article will discuss how to determine these interaction paths solely using PMU data.

\section{CONCLUSIONS}

This article has extended the results in Chompoobutrgool and Vanfretti (2012) aiming to further characterize the characteristics of "interaction paths" involved in swing dynamics of large-scale power systems. The concept of "secondary paths" was introduced, and the degree of persistence of these paths under different contingency conditions was shown.

Using the results from the contingency studies, it was shown that by knowledge of the properties of secondary paths, it is possible to make good choices to construct feedback signals for damping controllers. These signal combinations are relevant as they offer a back-up when loosing any of the signals from dominant paths that have the highest inter-area content Chompoobutrgool and Vanfretti (2012). This offers WAPODs increased flexibility by allowing feedback signal adaptation due to different failures.

\section{REFERENCES}

Chompoobutrgool, Y., Li, W., and Vanfretti, L. (2012). Development and Implementation of a Nordic Grid Model for Power System Small-Signal and Transient Stability Studies in a Free and Open Source Software. IEEE PES General Meeting.

Chompoobutrgool, Y. and Vanfretti, L. (2012). On the Persistence of Dominant Inter-Area Oscillation Paths in Large-Scale Power Networks. submitted to the $8^{\text {th }}$ IFAC PPEPSC Symposium.

Hauer, J., Trudnowski, D., and DeSteese, J. (2007). A perspective on WAMS analysis tools for tracking of oscillatory dynamics. IEEE PES General Meeting.

Kamwa, I., Beland, J., Trudel, G., Grondin, R., Lafond, C., and McNabb, D. (2006). Wide-Area Monitoring and Control at Hydro-Québec: Past, Present and Future. IEEE PES General Meeting.

Kundur, P. and et al. (2004). Definition and Classification of Power System Stability. IEEE Trans. on Pwr. Sys., 19(2), 1387-1401.

N.R. Chaudhuri, et al (2010). Wide-area power oscillation damping control in Nordic equivalent system. IET Gen., Trans. \& Distr, 4(10), $1139-1150$.

T. Van Cutsem (2010). Description, Modelling and Simulation Results of a Test System for Voltage Stability Analysis. Technical Report Version 1, IEEE WG on Test Systems for Voltage Stability Analysis.

Vanfretti, L. and Chow, J. (2009). Computation and Analysis of Power System Voltage Oscillations from Interarea Modes. IEEE PES General Meeting.

Vanfretti, L. and Chow, J. (2010). Analysis of Power System Oscillations for Developing Synchrophasor Data Applications. IREP Symposium - Bulk Power System Dynamics and Control.

Vanfretti, L. (2009). Phasor Measurement-Based State Estimation of Electric Power Systems and Linearized Analysis of Power System Network Oscillations. Ph.D. thesis, Rensselaer Polytechnic Institute, Troy, NY, USA.

Wei, X. (2002). Network Sensitivities of TCSC and SVC for Power Dispatching and Damping Control Design. Master's thesis, Rensselaer Polytechnic Institute. 